\title{
Pour en lire plus : Mutation : L'aventure humaine ne fait que commencer.
}

Cécile Redondo

\section{OpenEdition}

1 Journals

Édition électronique

URL : https://journals.openedition.org/ere/7664

ISSN : 2561-2271

Éditeur

Centr'ERE

Référence électronique

Cécile Redondo, «Pour en lire plus : Mutation : L'aventure humaine ne fait que commencer. »,

Éducation relative à l'environnement [En ligne], Volume 17-1 | 2022, mis en ligne le 27 janvier 2021 consulté le 02 février 2022. URL : http://journals.openedition.org/ere/7664

Ce document a été généré automatiquement le 2 février 2022

La revue Éducation relative à l'environnement est mise à disposition selon les termes de la Licence Creative Commons Attribution - Pas d'Utilisation Commerciale 4.0 International. 


\title{
Pour en lire plus : Mutation :
}

\section{L'aventure humaine ne fait que}

\section{commencer.}

\author{
Cécile Redondo
}

\section{RÉFÉRENCE}

Nathanaël Wallenhorst (2021). Mutation : L'aventure humaine ne fait que commencer. Paris : Le Pommier, 265 pages. 


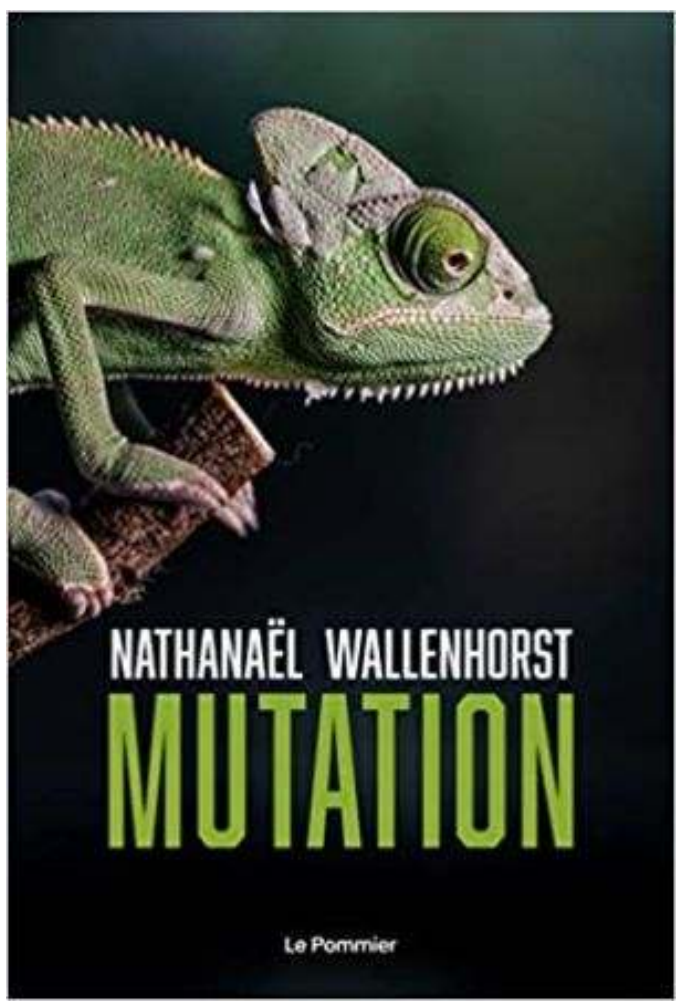

$1 \quad$ Mutation est un ouvrage qui théorise finement les concepts et processus en jeu au cœur de la nécessaire mutation humaine qu'il nous faut envisager en réponse à la mutation de la planète en cours. La transformation de l'humanité doit nécessairement prendre acte de la transformation en profondeur de notre monde, des limites de la Terre et de l'altération systématique du système Terre par les activités anthropiques. Nathanaël Wallenhorst ancre en effet son propos au sein de l'Anthropocène, "nouvelle époque géologique caractérisée par une modification durable des conditions d'habitabilité de la Terre pour l'ensemble du vivant » (p. 9), dont il est un expert en France, mais aussi dans la sphère internationale, en lien avec les nombreux ouvrages et articles qu'il a publiés sur le sujet. Face à ce processus de transformation de la Terre et de sa biosphère, un changement radical (au sens étymologique du terme radix « racine ») est nécessaire. La transformation en profondeur de l'humain doit s'exercer dans la façon dont nous vivons, dans la façon dont humains et non-humains coexistent (p. 8), mais aussi dans la façon dont nous nous considérons (p. 137) : « devenir autres que ce que nous sommes » c'est pour l'auteur l'« enjeu principal du xxI siècle » (p. 8). Il définit ainsi les contours et les possibilités de cette mutation politique et anthropologique fondée sur une relation avec le vivant (la biosphère).

2 Nathanaël Wallenhorst mène alors une enquête à travers les écrits scientifiques, sociologiques et philosophiques pour mettre au jour les soubassements de cette transformation profonde de l'humanité. L'auteur s'appuie par exemple sur la pensée des théoriciens critiques de la modernité (École de Francfort) et sur celle d'Hannah Arendt (p. 25-29), sur le concept de « résonance » d'Hartmut Rosa (p. 29-30, p. 44) et sur la notion de « limites planétaires » (et leurs transgressions) de Johan Rockström et coll. (p. 43). Le travail est richement étayé de nombreuses références théoriques dans le champ anthropo-politico-philosophique: Dominique Bourg, Christian Arnsperger, Andreas Weber, Jürgen Habermas, Ivan Illich, Maurice Merleau-Ponty, Günther Anders, Aristote, Descartes, etc. Ce cadre épistémologique est fondamental pour comprendre le 
développement de la thèse de l'auteur qui se résume ainsi: «seule une mutation anthropologique de nature à consolider le politique permettra à l'aventure humaine de se poursuivre » (p. 10). Tout au long de l'ouvrage, l'auteur précise les différents jalons de la thèse d'une anthropologie politique alternative à construire, de sorte que celle-ci s'enrichit au fil des cinq chapitres. Ceux-ci peuvent certes se lire séparément, mais gagnent assurément à être consultés globalement.

Il s'agit d'apprendre à faire société avec les non-humains (p. 14) et à coexister avec eux (p. 44) selon les principes du convivialisme post-prométhéen (p. 15-16, p. 44-46). Il est désormais nécessaire de penser dans le cadre d'une anthropologie de l'«immersion dans la nature » (p. 130-133, p. 137-138, p. 179, p. 191) et il s'agit aussi de rompre avec plusieurs écueils de la société moderne :

1. l'individualisme pour retrouver la nature « politique » de l'homme (p. 23);

2. le dualisme entre nature et culture pour penser les interdépendances entre l'humanité et la nature (p. 35, p. 136-138, p. 149, p. 178-180, p. 183);

3. l'anthropocentrisme pour renouveler notre rapport à la planète Terre en la replaçant au centre (p. 35-36, p. 44, p. 116);

4. le totalitarisme des Lumières - au sein desquelles s'enracine le développement technoindustriel, existant aujourd'hui dans la prédominance des calculs et algorithmes - pour repenser notre humanité renouvelée (p.37-38);

5. la segmentation des savoirs pour apprendre les savoirs de l'Anthropocène (p. 39).

L'«erreur fondamentale» se situe selon l'auteur du côté de l'anthropologie prométhéenne de la modernité (p. 21, p. 112) : cette mutation des transhumanistes qui consiste à améliorer ou augmenter la puissance humaine et à maitriser son environnement (p.20, p.44, p.116, p.140-141, p. 234) en appui au capitalisme, au néolibéralisme (p. 8, p. 145) et à la technocratie (via la quête infinie du progrès de la science et le "génie technoscientifique ", p. 110). Pour Nathanaël Wallenhorst, l'enjeu de l'entrée en Anthropocène est que celle-ci «est révélatrice d'une problématique anthropologique dans le rapport à la limite» (p. 114). L'auteur articule alors autour de ce concept central de l'Anthropocène sa vision critique argumentée du paradigme transhumaniste, qui s'avère convaincante :

1. les théories et recherches transhumanistes, en renforçant les problématiques écologiques, repoussent encore les limites environnementales et anthropologiques plutôt que de les accepter et les intégrer (p. 143, p. 149-150, p. 160);

2. le transhumanisme, en mettant l'accent sur la mutation de l'individu, est un projet qui évince le politique alors que le «centre de gravité » de la mutation humaine à envisager est « la relation » et pas l'individu (p. 119, p. 149).

5 Nathanaël Wallenhorst nous invite pas à pas à découvrir les différents «manifestes » et " plaidoyers » de ces dix dernières années (p. 14-18) pour mettre au jour la façon dont ils mobilisent le concept d'Anthropocène et participent à sa montée en puissance en politique : c'est l'objet du chapitre II. Sont ainsi examinés neuf textes majeurs produits au cours de cette dernière décennie : le Manifeste des économistes atterrés $(2010,2015)$, le Manifeste accélérationniste (2013), le Manifeste écomoderniste (2015), le Manifeste pour l'Anthropocène (2015), le Manifeste des communs (2018), le Manifeste animaliste (2017), le Manifeste pour une justice climatique (2019), le Manifeste de l'Écologie intégrale (2019) et le Manifeste convivialiste (2013). À travers la présentation et l'analyse de ces manifestes, l'auteur montre comment ils pointent « un problème fondamental dans notre relation au monde» et postulent "autant la nécessité de muter que de repenser l'aventure 
humaine » (p. 108). Le chapitre III est quant à lui consacré à caractériser cette mutation anthropologique plus ou moins présente (sous une forme ou une autre) dans chacun des manifestes: une mutation humaine susceptible de consolider le politique. Puis l'auteur en propose dans le chapitre IV une modélisation: l'« aventure humaine" comme mutation politique.

Enfin, Nathanaël Wallenhorst, en investissant la question de l'éducation, l'identifie comme le moyen de la mutation anthropologique qu'impose l'Anthropocène (p. 40). Mais c'est aussi une éducation au politique visant l'apprentissage de la responsabilité du monde (p. 45). Cette dimension transversale à l'ouvrage (pp. 38-41) est également abordée dans le cinquième et dernier chapitre qui pose la question de comment accompagner et comment mettre en œuvre concrètement la mutation humaine requise par l'Anthropocène. Selon l'auteur, le niveau éducatif relève bien d'une radicalité " convivialiste et démocratique » (p. 199). Dans sa démarche d'appréhension de l'action éducative, Nathanaël Wallenhorst utilise une métaphore centrale que nous résumons ainsi : face à la guerre engagée, éducateurs et apprenants doivent apprendre à se battre et mener le combat en utilisant les armes du droit, du savoir et de la démocratie (p. 200-203). La finalité est l'apprentissage de l'amour du monde et de la Terre à travers les apprentissages politiques de l'hospitalité et de la responsabilité ; un des moyens est d'apprendre à écouter (préalable à apprendre à répondre).

7 En conclusion, le livre de Nathanaël Wallenhorst aborde avec pertinence et originalité la thématique actuelle de l'Anthropocène au prisme de la mutation humaine qui lui est consubstantielle. Nourri par des éléments théoriques et une vaste littérature sur le sujet ainsi que par une analyse du corpus des plus grands manifestes contemporains, il offre des éclairages importants aux étudiants et aux chercheurs sur la notion, et plus généralement sur le champ de l'éducation en Anthropocène. Grâce à cet ouvrage, les chercheurs et praticiens de l'éducation relative à l'environnement qui s'intéressent à l'Anthropocène trouveront des cadres, des outils et des méthodes pour explorer de façon rigoureuse nos relations - vivantes, résonantes, conviviales - aux humains, aux non-humains et au monde. Nathanaël Wallenhorst ouvre ainsi la porte à des recherches contemporaines portant sur des dispositifs et situations à mettre en œuvre permettant de contribuer à cette mutation en contextes éducatifs et formatifs.

\section{AUTEUR}

\section{CÉCILE REDONDO}

Chercheure post-doctorante, Université Aix-Marseille - courriel : cecile.REDONDO[@]univ-amu.fr 